\title{
The Effect of Six-Week HIIT Swimming Exercise and Resveratrol Supplementation on the Level of SIRT3 in Frontal Lobe of Aged rats
}

\author{
Amin Mehrabi ${ }^{1,2}$, Abbasali Gaeini ${ }^{3}$, Reza Nouri $^{*}$, Farhad Daryanoosh ${ }^{4}$ \\ ${ }^{1}$ Department of Sport Sciences, Kish International Campus, University of Tehran, Kish, Iran \\ ${ }^{2}$ Neuroscience Research Center, Institute of Neuropharmacology, Kerman University of Medical Sciences, Kerman, Iran \\ ${ }^{3}$ Department of Exercise Physiology, Faculty of Physical Education, University of Tehran, Tehran, Iran \\ ${ }^{4}$ Department of Exercise Physiology, Faculty of Educational Sciences and Psychology, Shiraz University, Shiraz, Iran
}

Article Info:

Received: 15 Aug 2020

Revised: 10 Nov 2020

Accepted: 15 May 2021

\section{A BSTRACT}

Introduction: Mitochondrial disorders play an essential role in reducing the health, infestation, and progression of aging. The SIRT3 in mitochondria coordinates many mitochondrial biological aspects that are important in aging. Moreover, it alters directly the activity of many metabolic enzymes. Exercise has been able to enhance SIRT3 protein's expression and improve antioxidant function and neuroprotection. Resveratrol acts as a modulator of SIRT3 and has anti-aging and neuroprotective effects. The aim of this study was to determine the effect of HIIT swimming activity and resveratrol supplementation on SIRT3 levels in the frontal lobe among older rats. Materials and Methods: Thirty rats were randomly divided into 5 groups; control (C), solvent (S), supplement (R), HIIT exercise (EX), HIIT exercise, and supplement (EXR)). The EX group performed HIIT swimming training for six weeks. Group $\mathrm{C}$ rats did not practice. Group R rats received only resveratrol supplementation. Rats in the EXR group performed HIIT swimming exercises with resveratrol. Group S received the only solvent. The SIRT3 protein levels were assessed frontal lobe of the rats. Results: A significant increase in SIRT3 protein was observed in group R, EX, and EXR groups compared to the control group. In addition, there was a substantial difference between the mean values of SIRT3 protein among the three groups. SIRT3 levels in the EX and EXR groups were significantly greater compared to the R group. Conclusion: Increasing the amount of SIRT3 in the response of HIIT swimming training in older rats indicating the involvement of this protein in metabolic pathways, antioxidant defense, and neuroprotection. In addition to metabolic tissues, this process occurs in the frontal lobe of the brain.

\section{Keywords:}

1. Resveratrol

2. Swimming

3. Aging

*Corresponding Author: Reza Nouri

Email: nuri_r7@ut.ac.ir 


\title{
تأثير شش هفته فعاليت ورزشى HIIT شنا و رزوراترول بر ميزان SIRT3 در لوب بيشانى مغز موشهاى صحرايى وِير برون
}

\author{
امين مهر ابى “'، عباسعلى كَائينى"، رضا نورى"، فرهاد دريانوش" \\ اكروه علوم ورزشى، برديس كيش، دانشكاه تهران، كيش، ايران

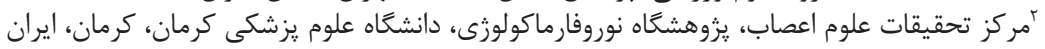

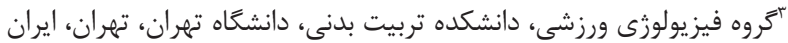

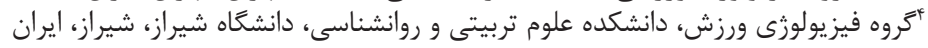

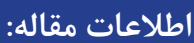

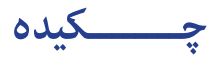

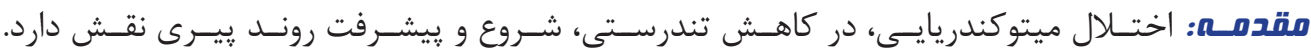

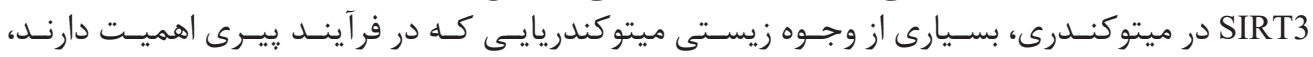

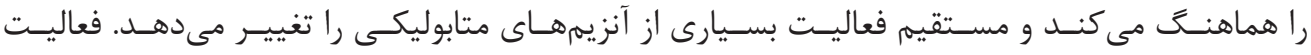

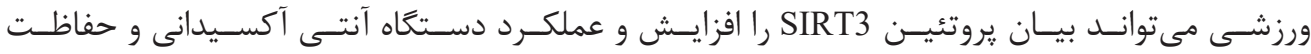

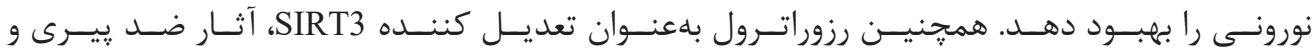

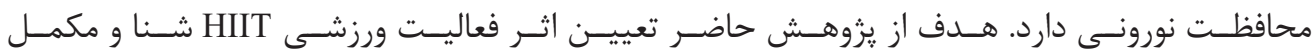

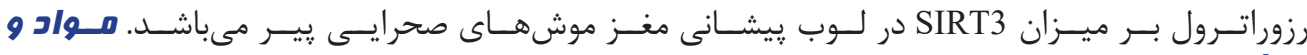

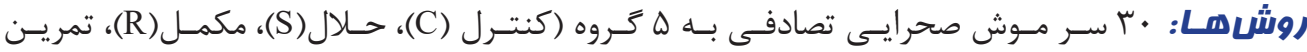

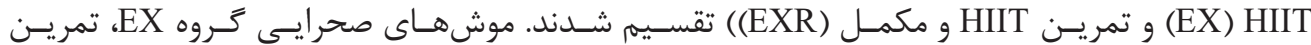

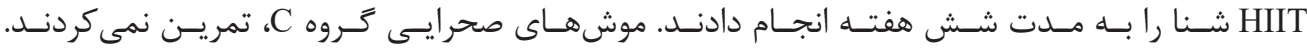

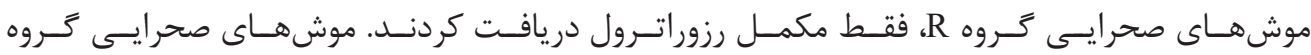
EXR

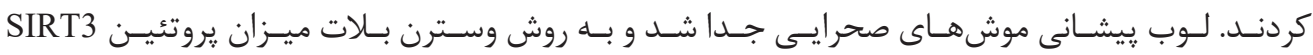

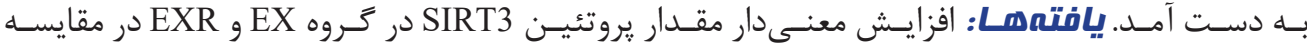

وازههاى كليدى: (- ماز (ب

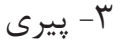

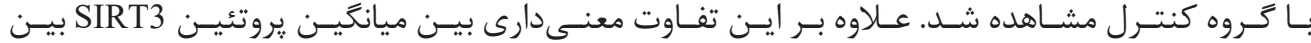

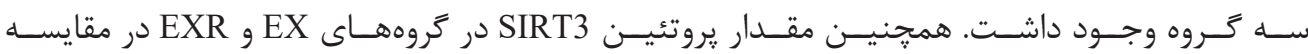

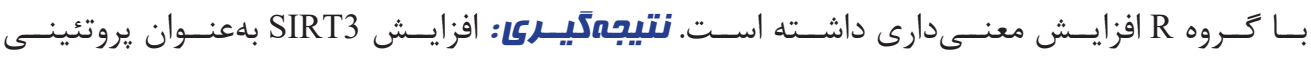

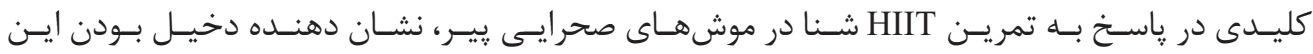

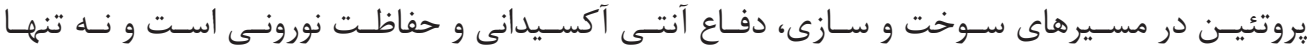

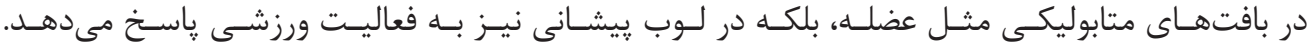




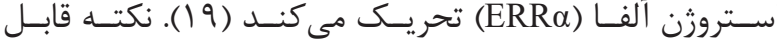

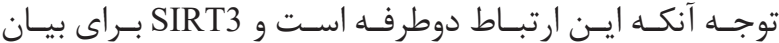

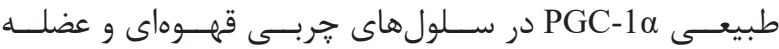

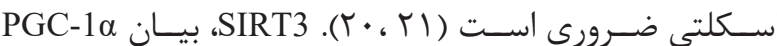

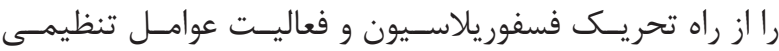

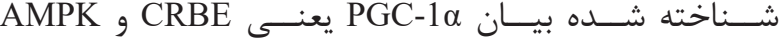

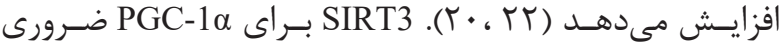

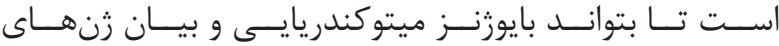

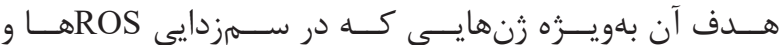

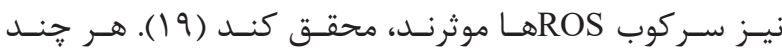

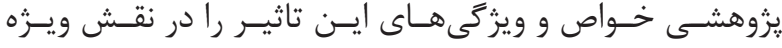

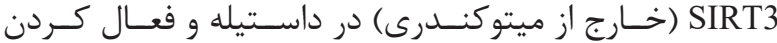

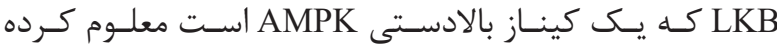

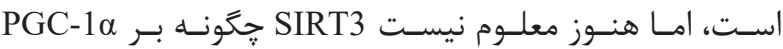

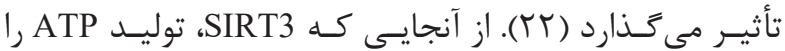

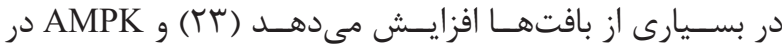

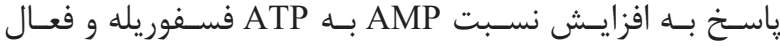

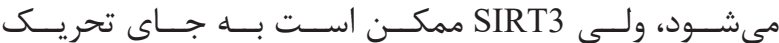

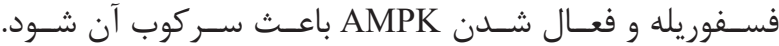

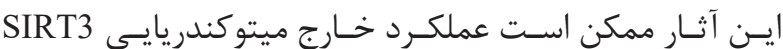

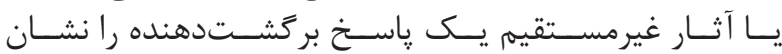

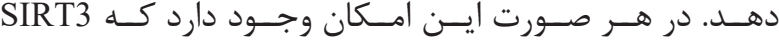

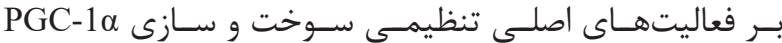

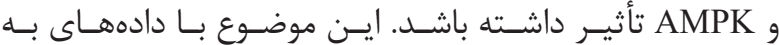

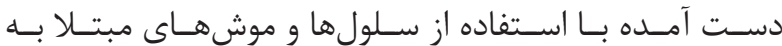

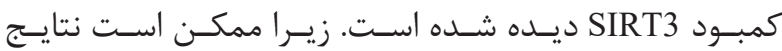

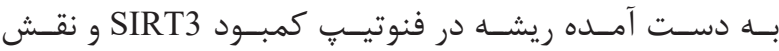

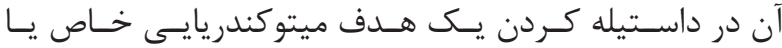

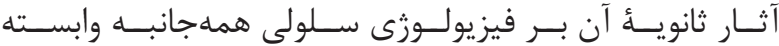

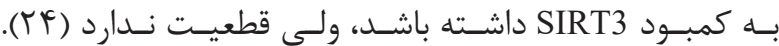

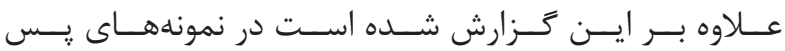

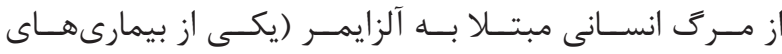

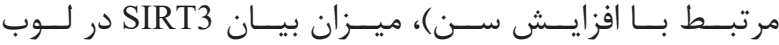

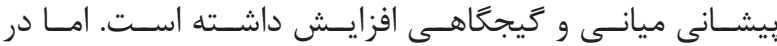

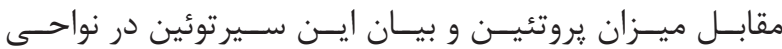

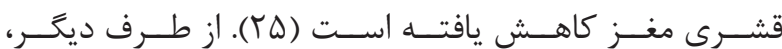

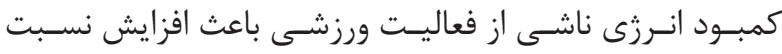

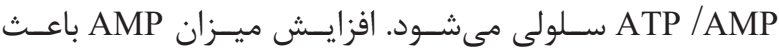

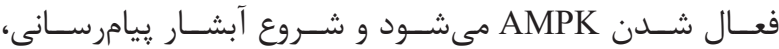

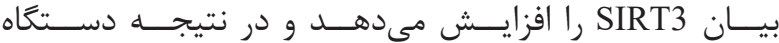

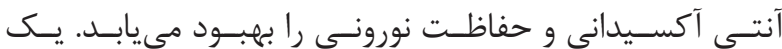

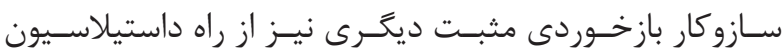

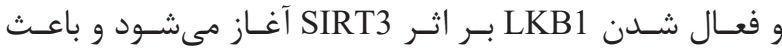

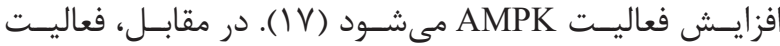

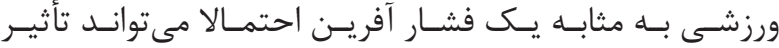

مقلمهه

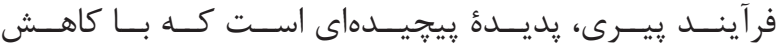

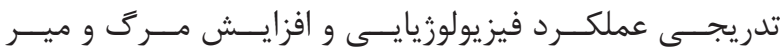

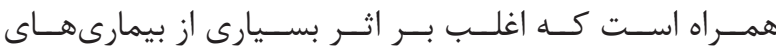

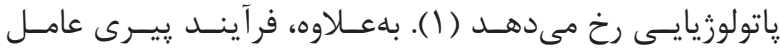

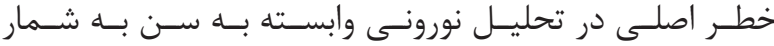

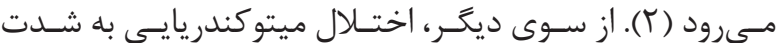

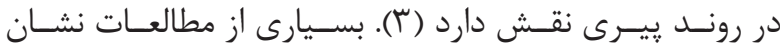

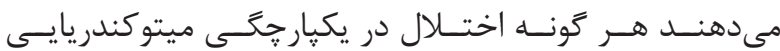

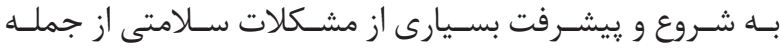

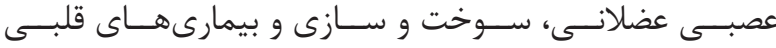

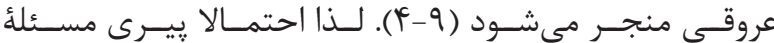

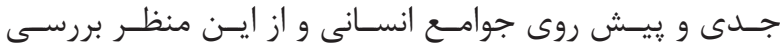

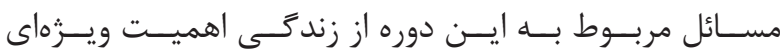

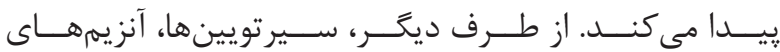

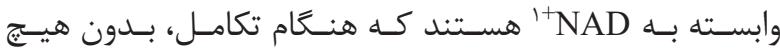

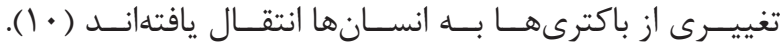

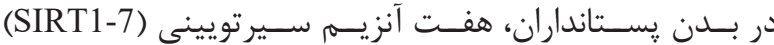

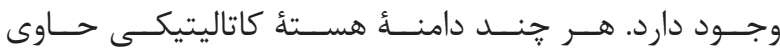

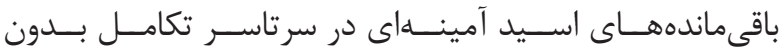

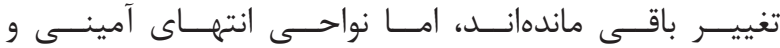

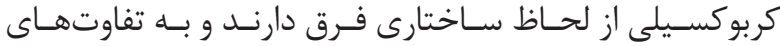

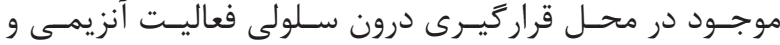

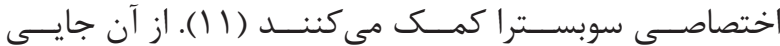

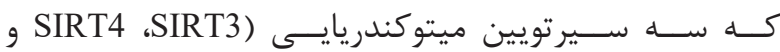

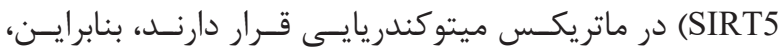

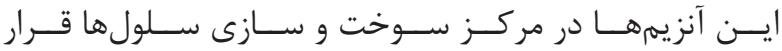

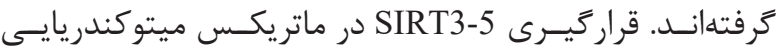

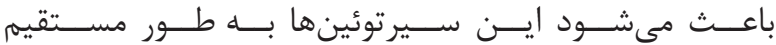

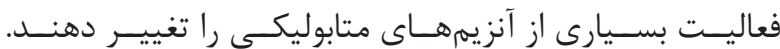

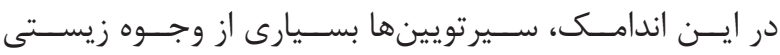

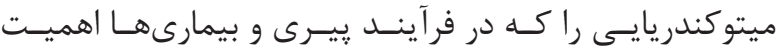

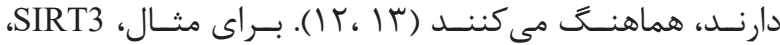

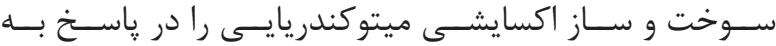

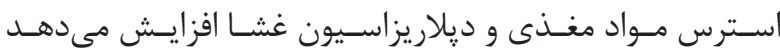

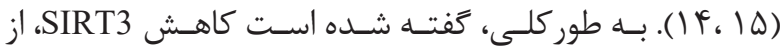

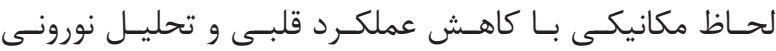

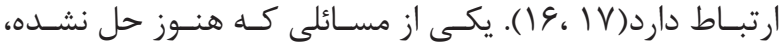

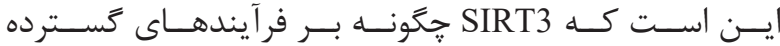

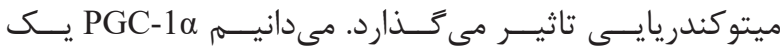

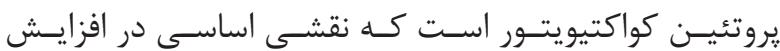

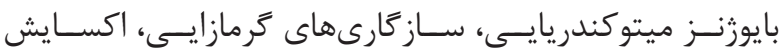

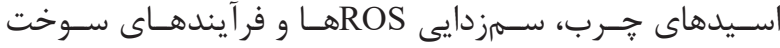

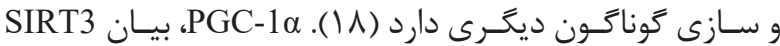

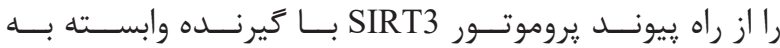




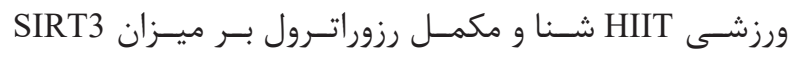

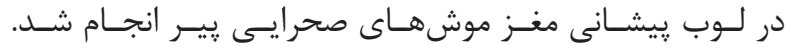

\section{مواد و روشها}

يزوهــش حاضــر، از نــوع توسـعلهاى اسـت و از نظـــر روش كار

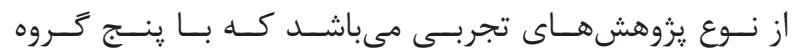

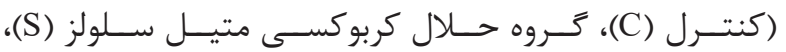

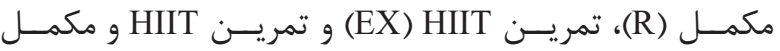

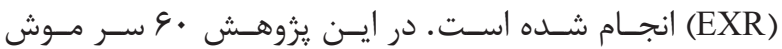

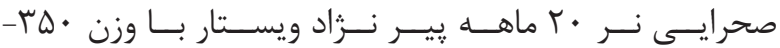

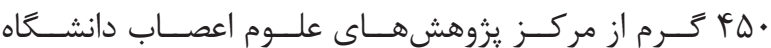

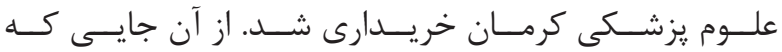

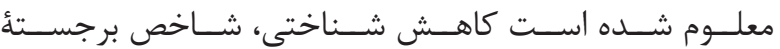

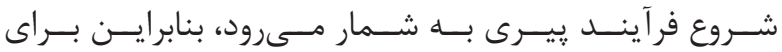

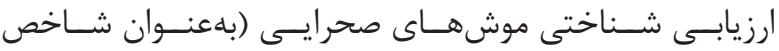

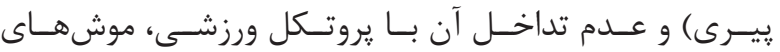

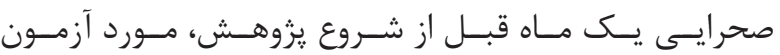

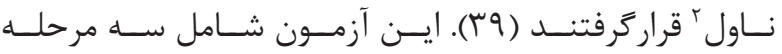

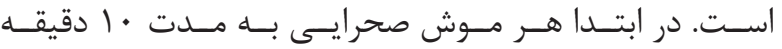

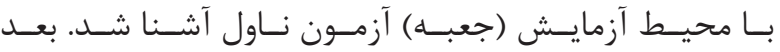

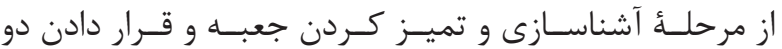

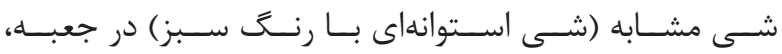

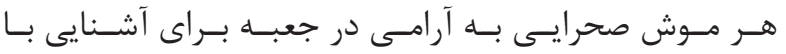

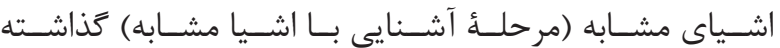

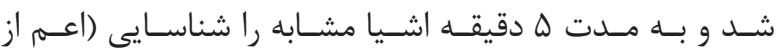

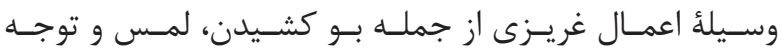

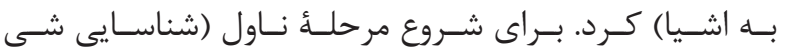

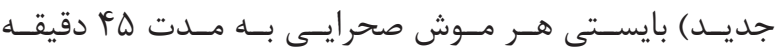

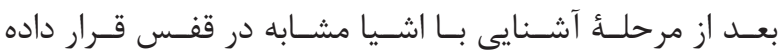

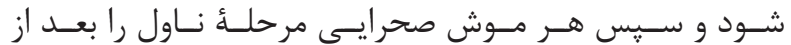

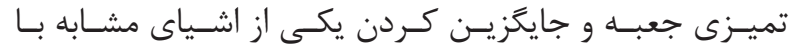

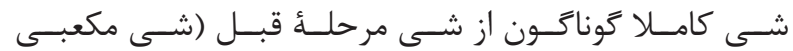

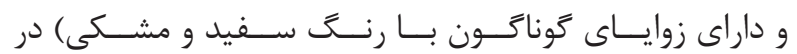

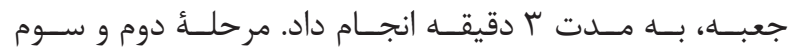

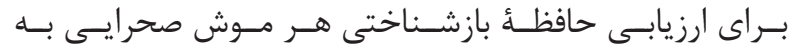

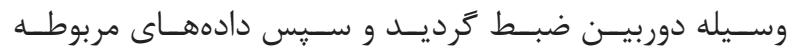

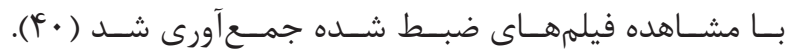

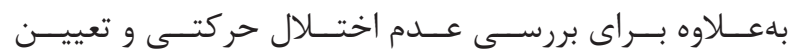

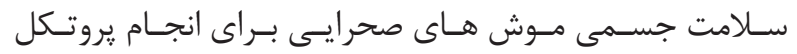

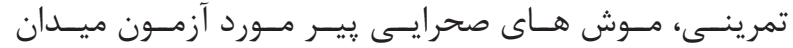

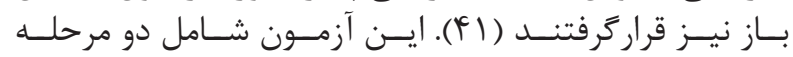

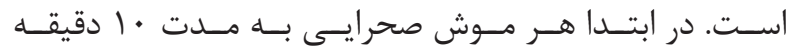

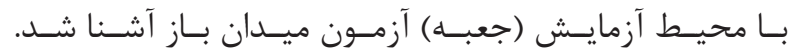

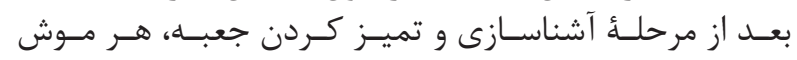

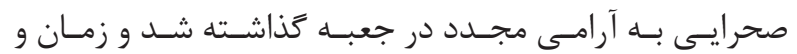

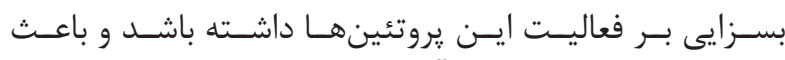

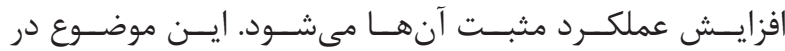

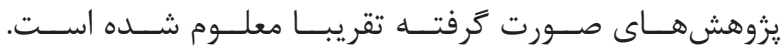

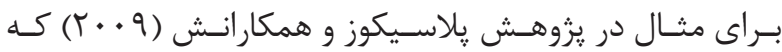

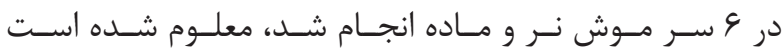

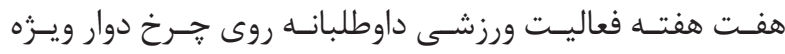

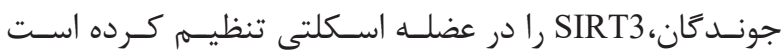

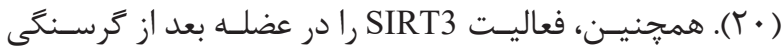

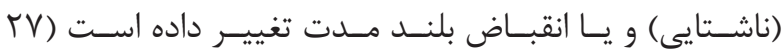

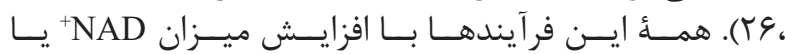

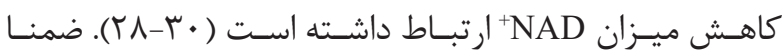

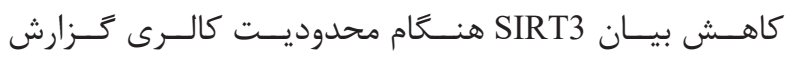

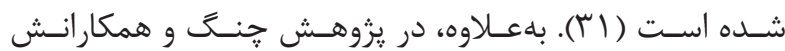

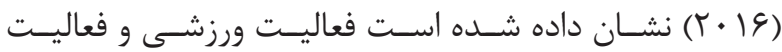

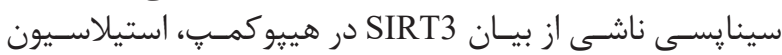

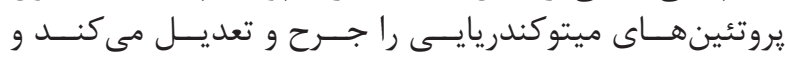

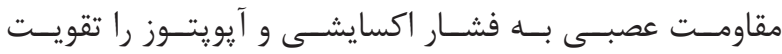

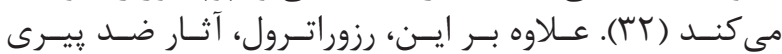

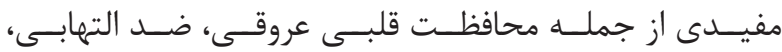

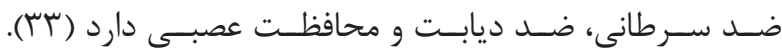

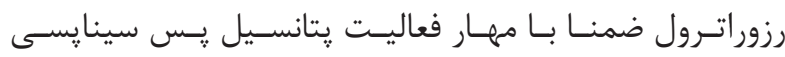

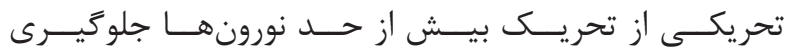

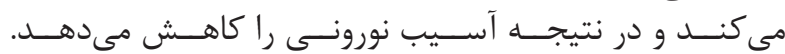

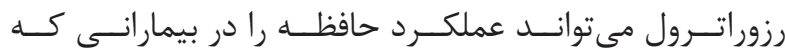

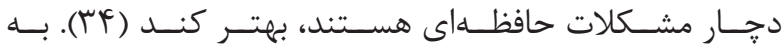

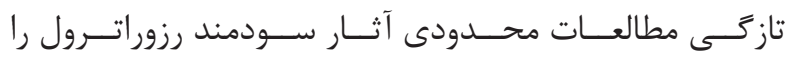

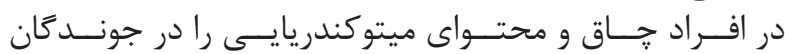

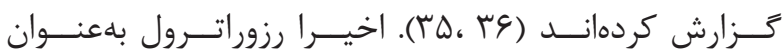

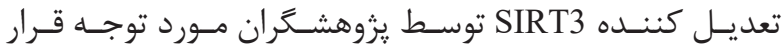

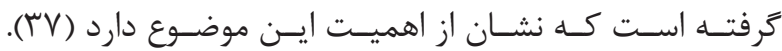

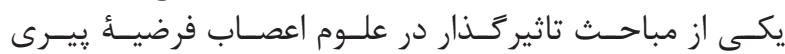

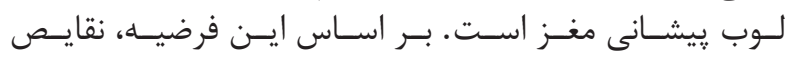

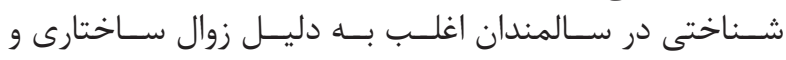

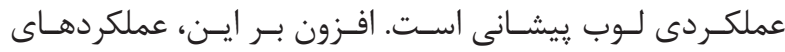

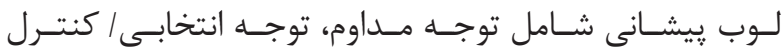

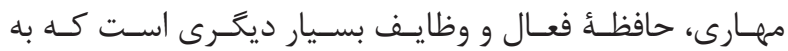

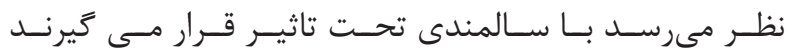

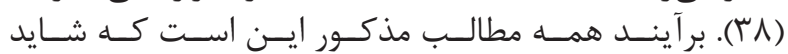

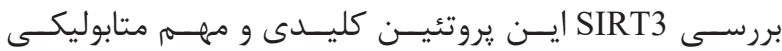

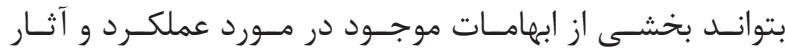

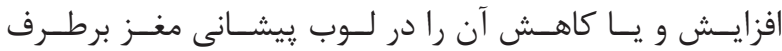

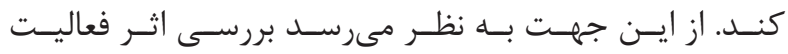

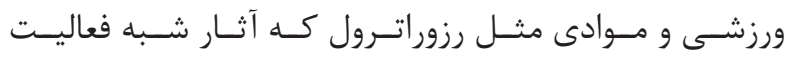

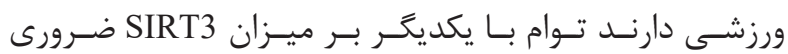

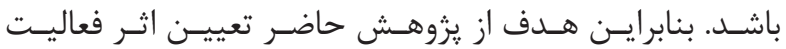




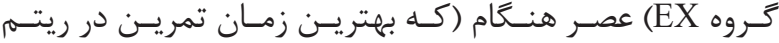

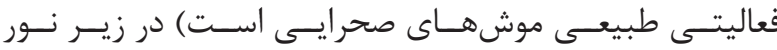

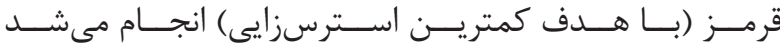

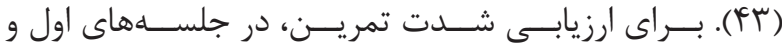

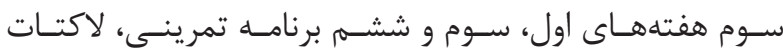

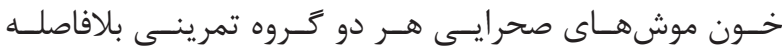

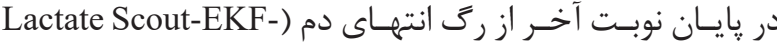

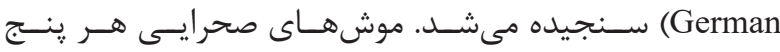

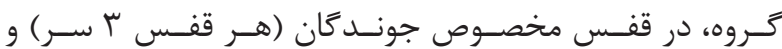

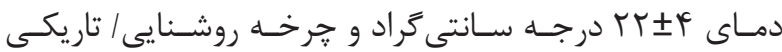

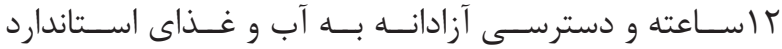

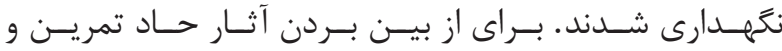

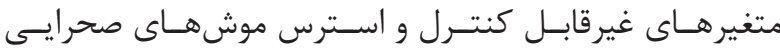

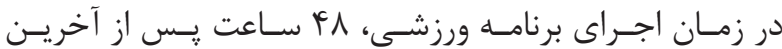

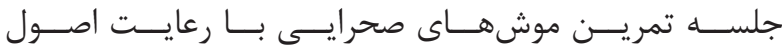

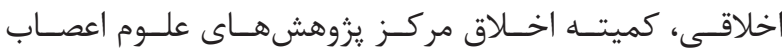

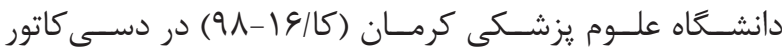

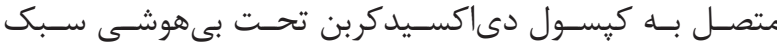

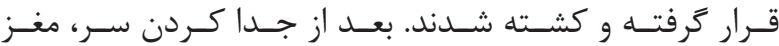

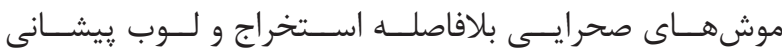

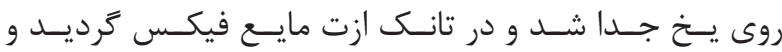

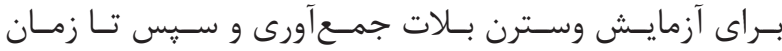

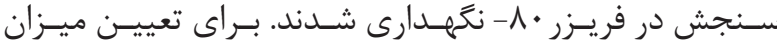

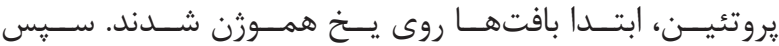

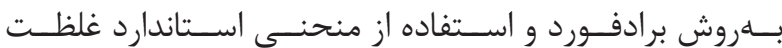

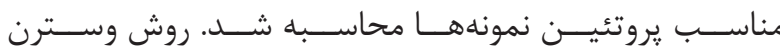

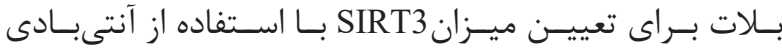

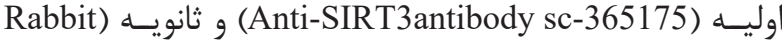
: (6c5) GAPDH , (anti-mouse IgG-HRP:sc-358914

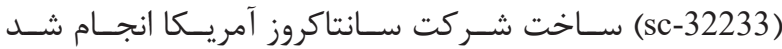

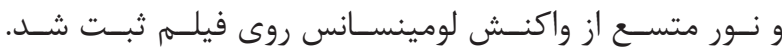

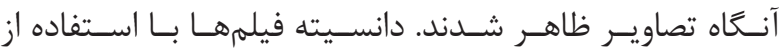

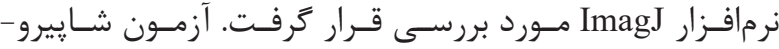

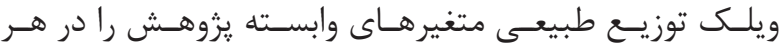

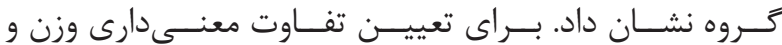

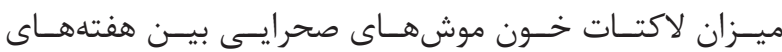

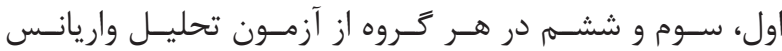

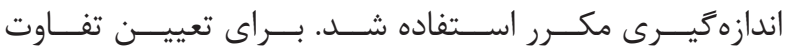

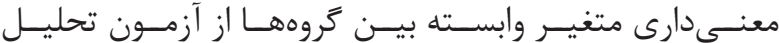

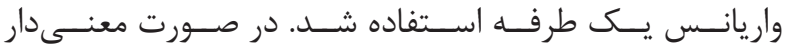

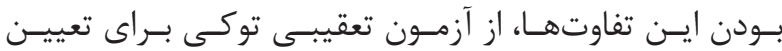

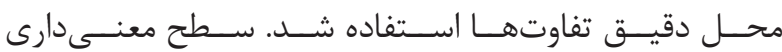

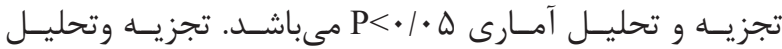

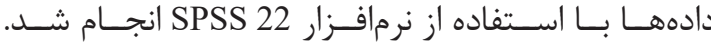

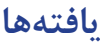

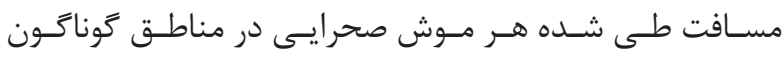

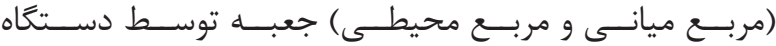

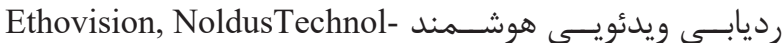
دogy, version7

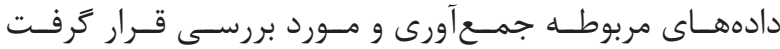

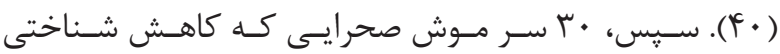

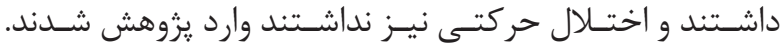

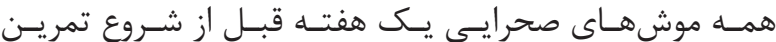

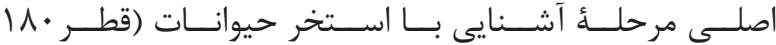

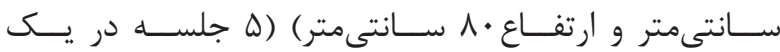

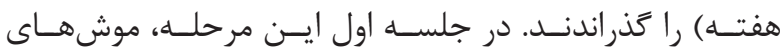

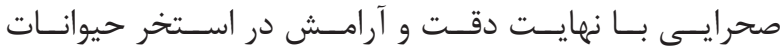

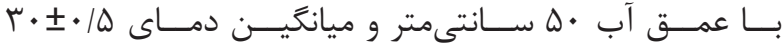

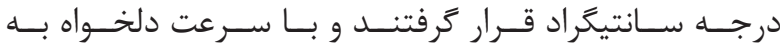

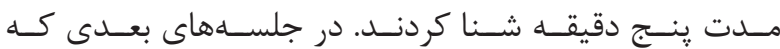

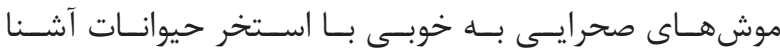

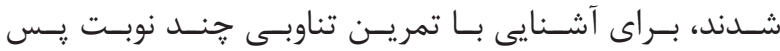

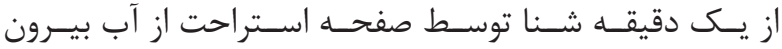

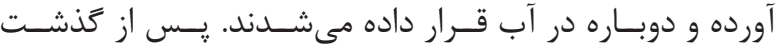

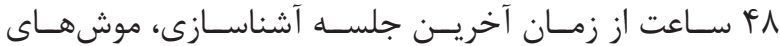

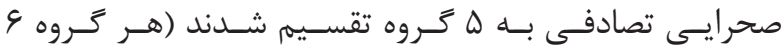

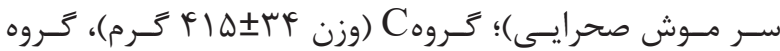
EX EXR

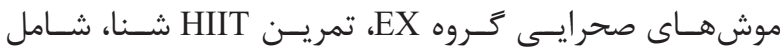

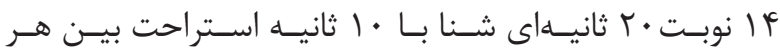

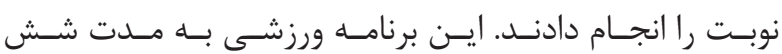

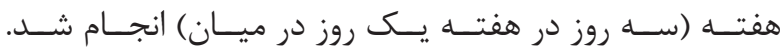

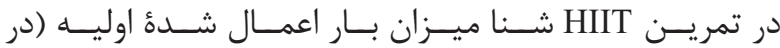

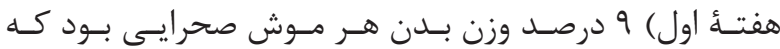

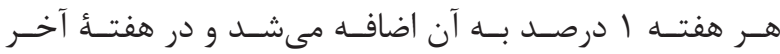

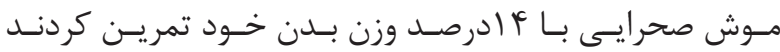

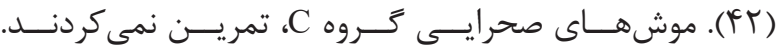

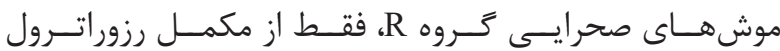

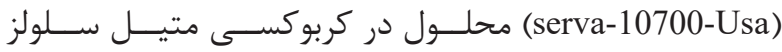

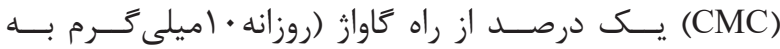

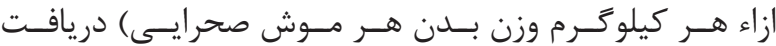

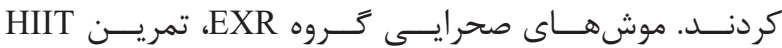

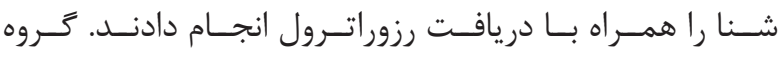

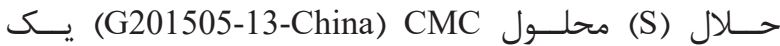

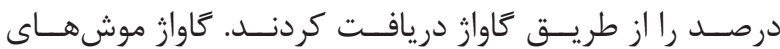

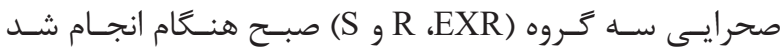

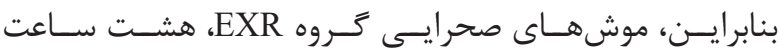

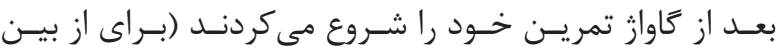

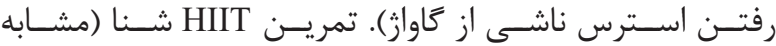




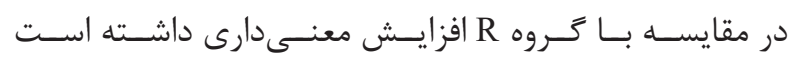

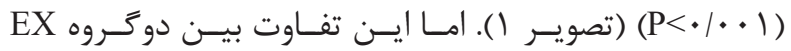

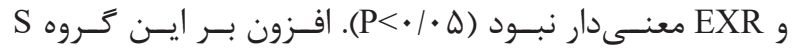

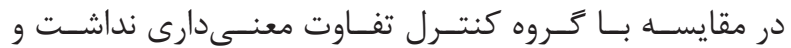

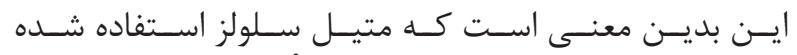

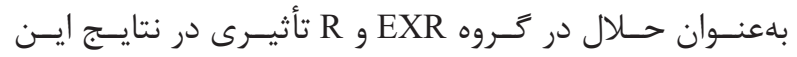

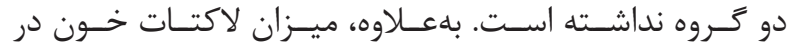

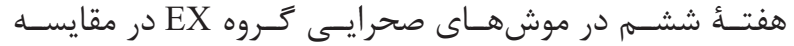

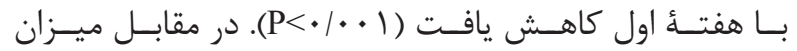

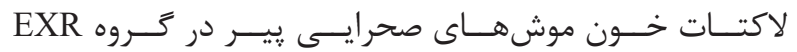

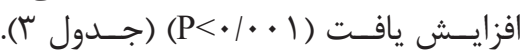

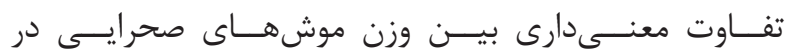

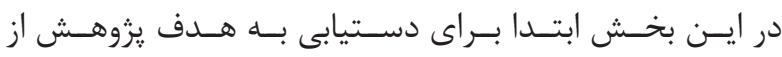

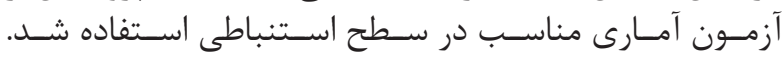

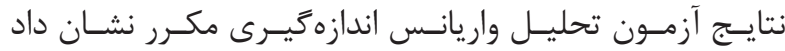

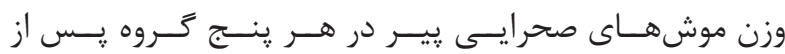

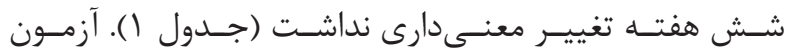

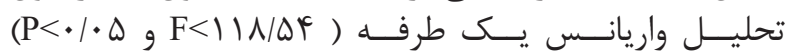

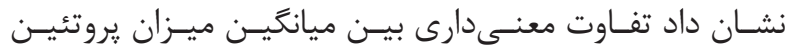
SIRT3

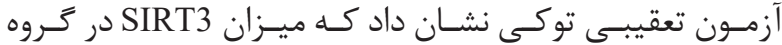

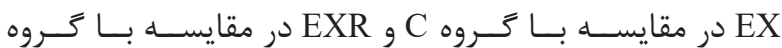

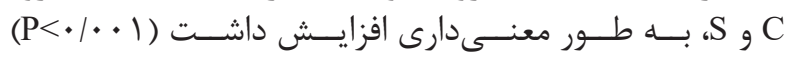

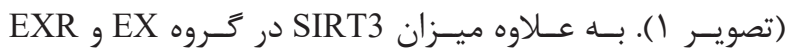

جدول ا- ميانكَين وزن موشهاى صحرايى در هفتههاى اول، سوم و ششم

\begin{tabular}{|c|c|c|c|c|}
\hline انحراف استاندارد & ميانين & ت تعداد & تروه & هفته \\
\hline$r 4 / 19$ & $F \mid \Delta / \& Y$ & \& & كت-رل & \multirow{5}{*}{ اول } \\
\hline$r 1 / 90$ & F. ITT & c & حالال & \\
\hline$r \cdot 119$ & h../tt & 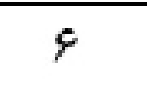 & مكمل & \\
\hline$r 1 / 09$ & $F \cdot I / F F$ & c & تمرين و مكمل & \\
\hline$\Gamma \cdot / 9 \lambda$ & $f \cdot 1 / 4 f$ & 9 & تمرين & \\
\hline$r 1 / \cdot \varphi$ & FI9/T & 8 & كتبرل & \multirow{5}{*}{ سوم } \\
\hline rs/दq & $f\|\|$ & \& & حلال & \\
\hline THIAT & rarflet & $q$ & مكمل & \\
\hline$T \Delta / T$. & raf/Tr & 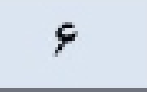 & تمرين و مكمل & \\
\hline$T A / F T$ & $F \cdot G / T T$ & c & تمرين & \\
\hline$r \Delta / T)$ & FTQ/DG & 9 & كنترل & \multirow{5}{*}{ شُمه } \\
\hline אד/Tr & FTV/Aq & 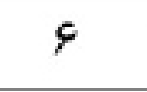 & حلال & \\
\hline re/gV & rq. $19 y$ & 4 & مكمل & \\
\hline $\mid \mathrm{V} / \cdot \mathrm{r}$ & 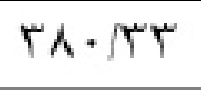 & 9 & تمرين و مكمل & \\
\hline ५१/१८ & $F \cdot \Delta / T T$ & $c$ & تمرين & \\
\hline
\end{tabular}




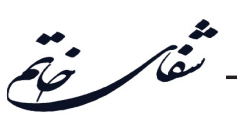

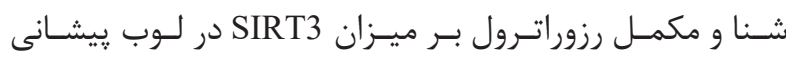

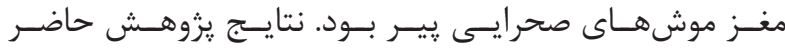

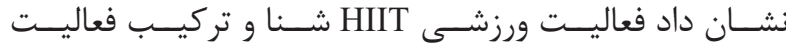

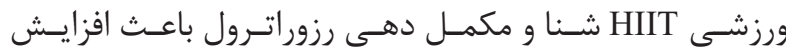

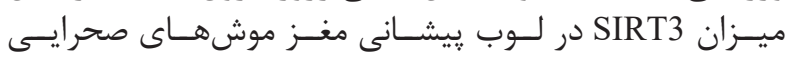

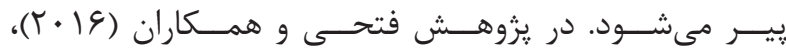

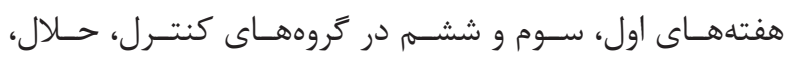

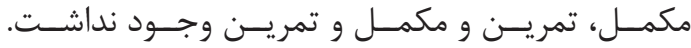
*فاوت معنى دارى در مقايسه با هفتئ اول بحث و نتيجه

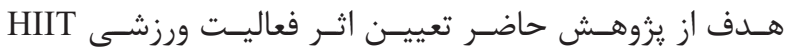

جدول r- نتايج آزمون تحليل واريانس يك طرفه SIRT3 ناشى از فعاليت ورزشى HIIT شنا و رزوراترول

\begin{tabular}{|c|c|c|c|c|c|}
\hline معنى مارى & $\mathbf{F}$ & ميانگين مجذورات & درجه آزادى & مجموع مجذورات & SID? \\
\hline$\cdot 1 \cdot \cdot 1$ & $\| N / \Delta F$ & $1 / \cdot 9$ & f & $r / r \Delta \Lambda$ & \\
\hline
\end{tabular}

جدول بـ- ميانگَين تغييرات لاكتات خون موشهاى صحرايى بير در هفتههاى اول، سوم و ششم تمرين HIIT شنا

\begin{tabular}{|c|c|c|c|c|}
\hline هفتهُ ششمر & هفتهُ سوم & هفتهُ اول & ترووها & متغير \\
\hline$r / \cdot 1 \pm \cdot / 1 r$ & $r / \cdot \pm \pm \cdot / 1 \mathrm{~V}$ & $r / \cdot r \pm \cdot / 1 Q$ & $\mathrm{C}$ & \\
\hline$* q / 11 \pm \cdot / r q$ & $\Lambda / \Lambda \cdot \pm \cdot|Q|$ & $\Lambda / \& \Lambda \pm 1 / \& \Lambda$ & EX & \\
\hline$* 1.1 \cdots \pm \cdot / 4 \Lambda$ & $* q / \Lambda \cdot \pm \cdot / r v$ & $N / Q \cdot \pm 1 / v 9$ & EXR & ميلىمول/ ليتر \\
\hline
\end{tabular}

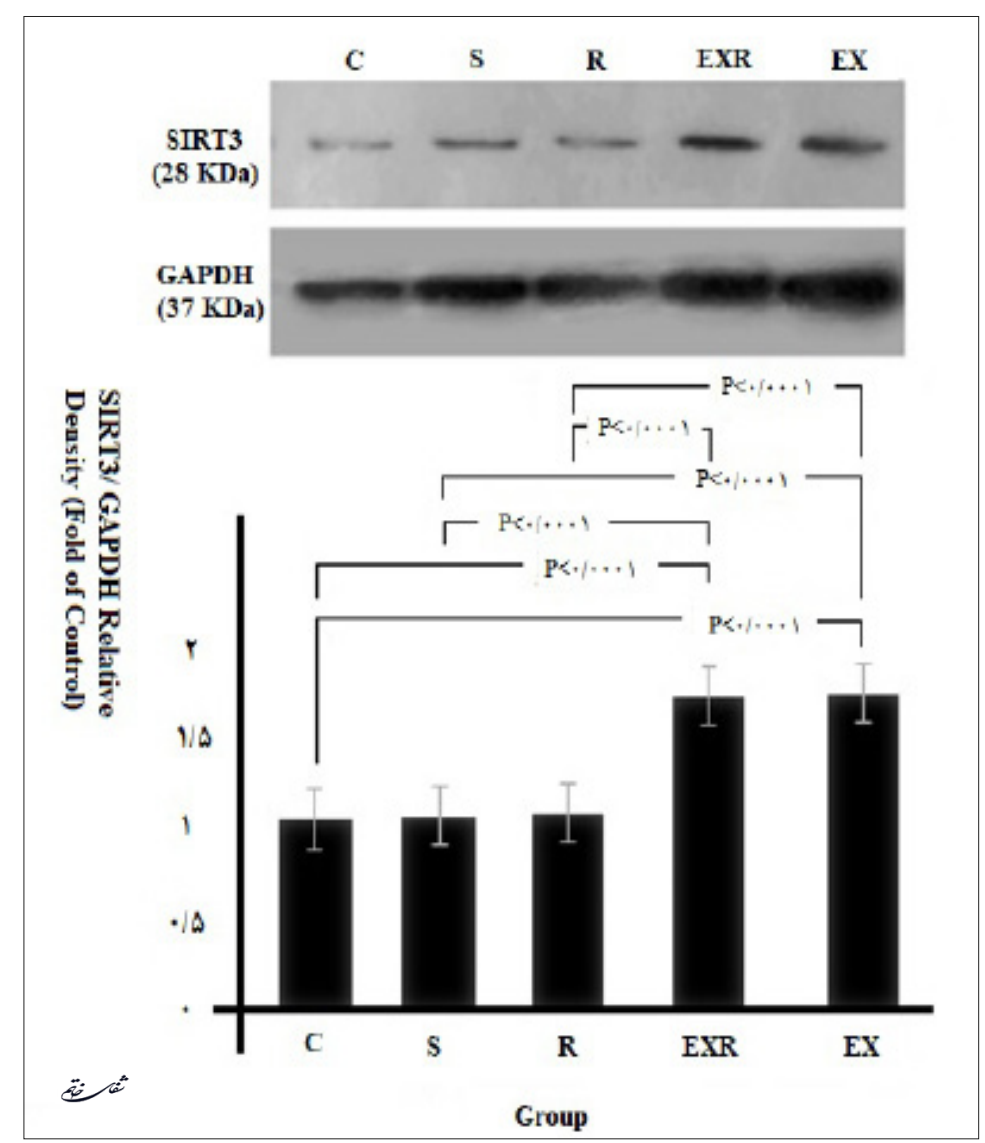

تصوير ا- مقايسٔ ميزان يروتئين SIRT3/GAPDH بين گروههاى سطح معنى دارى براى همد گروهها هـ/• در نظر خرفته شد. 


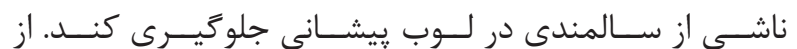

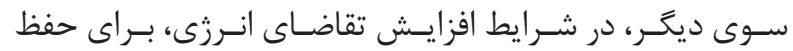

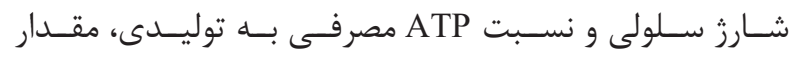

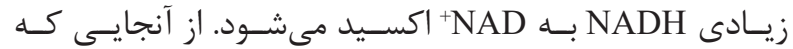

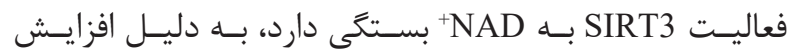

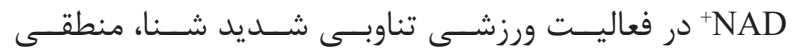

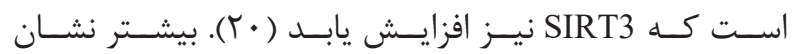

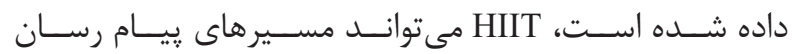

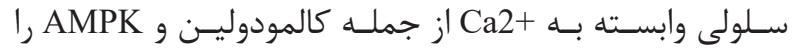

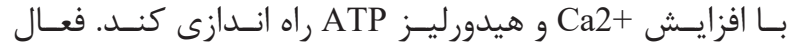

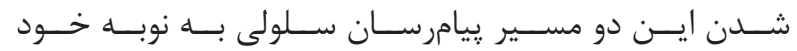

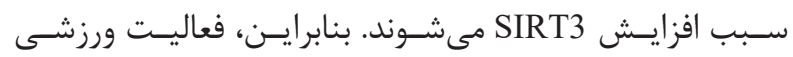

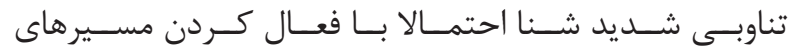

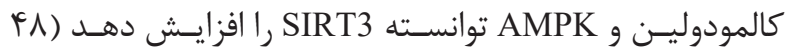

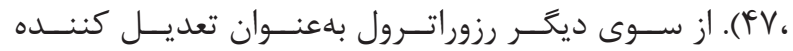

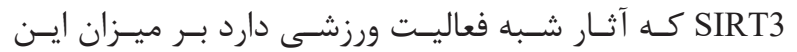

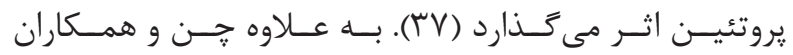

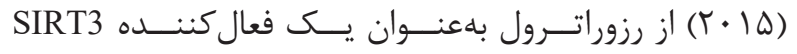

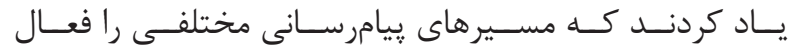

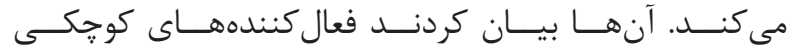

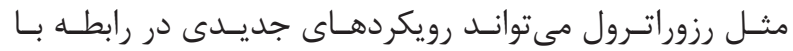

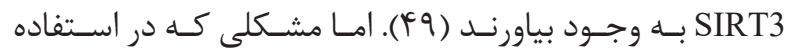

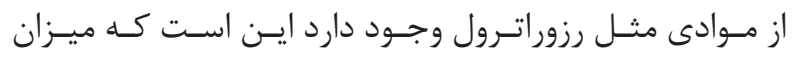

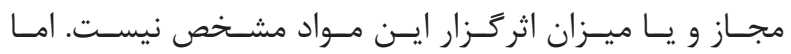

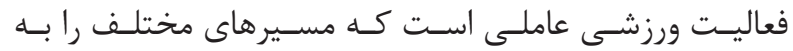

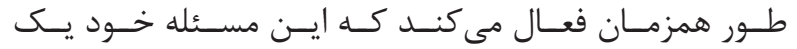

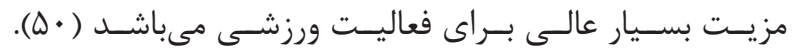

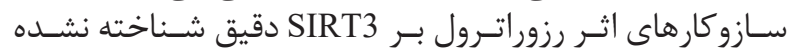

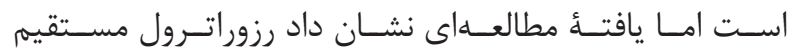

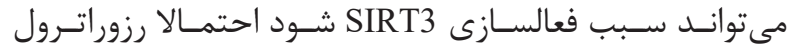

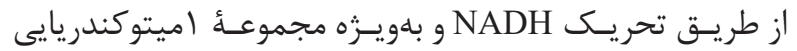

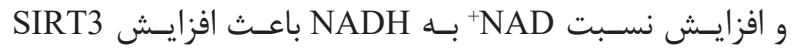

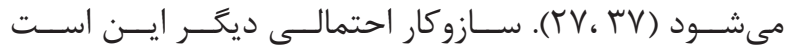

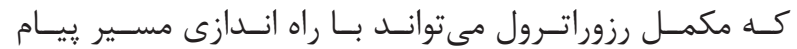

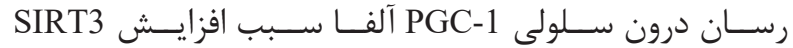

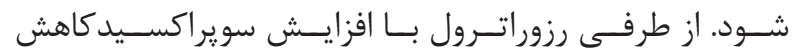

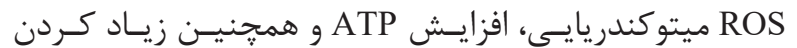

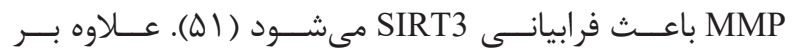

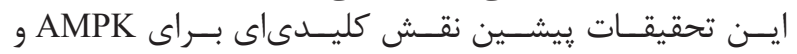

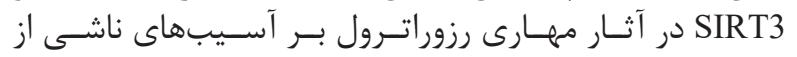

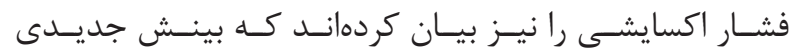

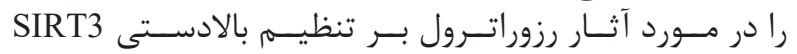

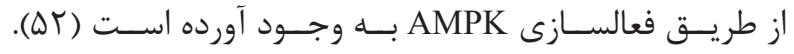

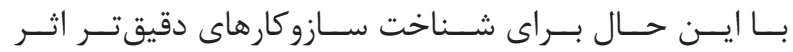

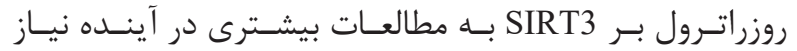

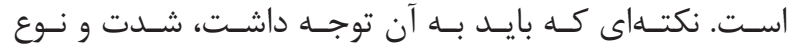

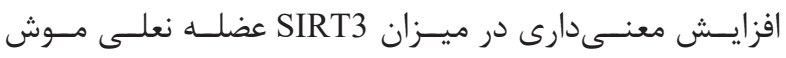

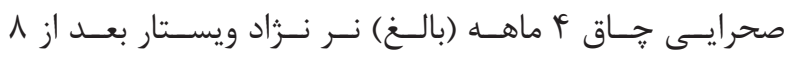

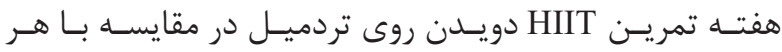

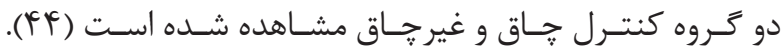

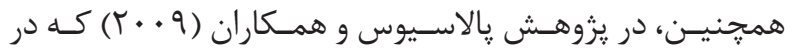

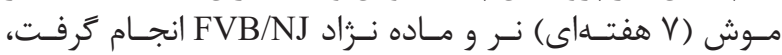

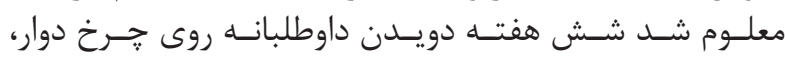

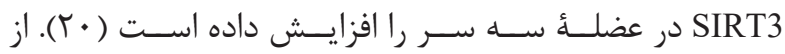

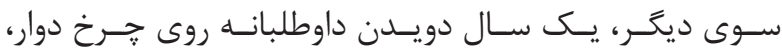

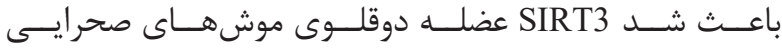

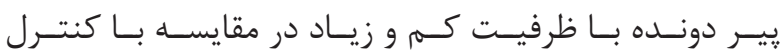

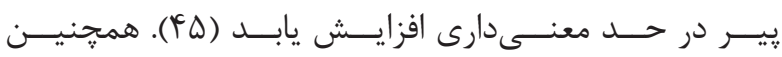

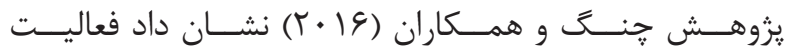

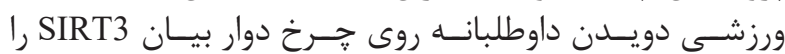

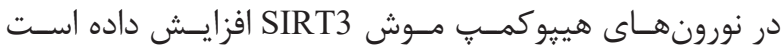

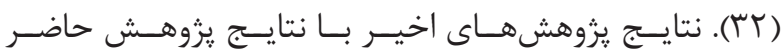

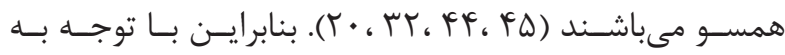

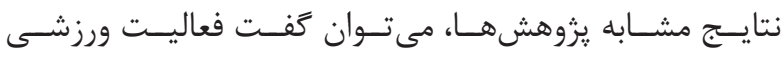
HIIT

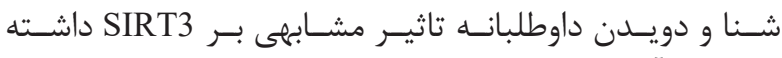

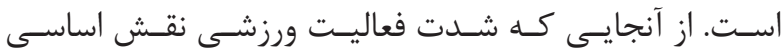

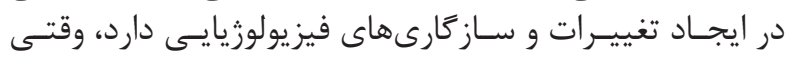

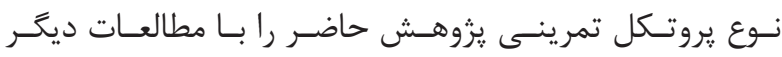

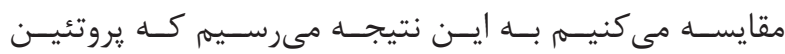

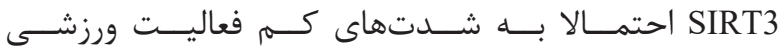

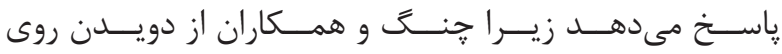

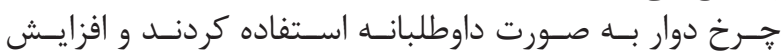

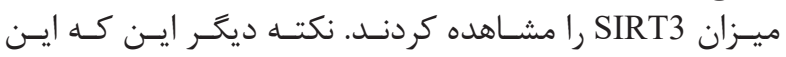

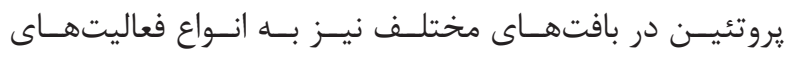

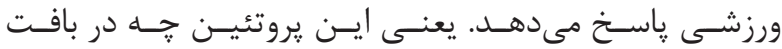

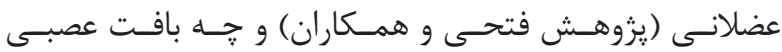

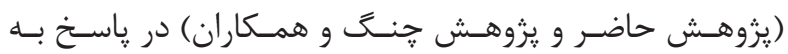

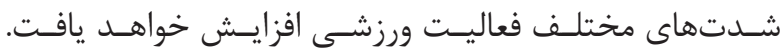

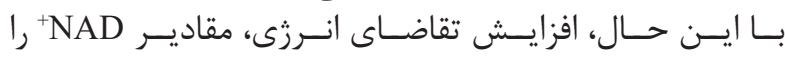

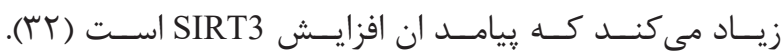

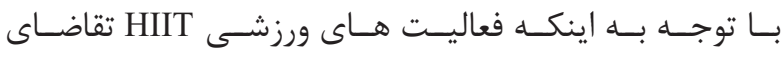

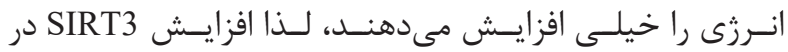

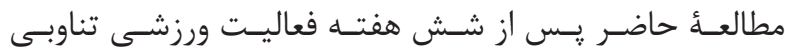

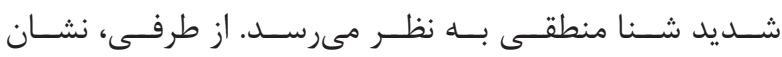

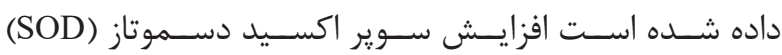

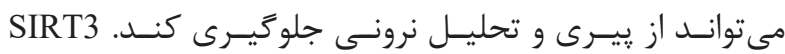

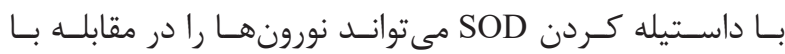

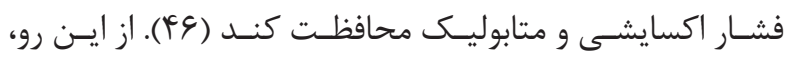

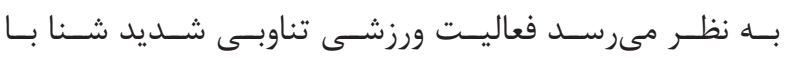

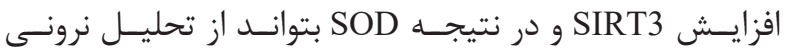




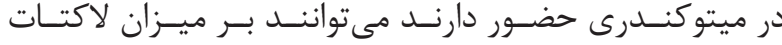

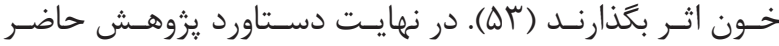

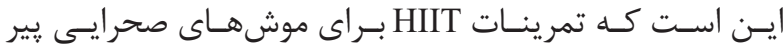

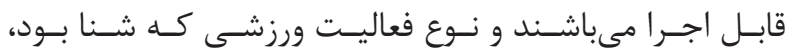

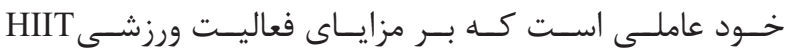

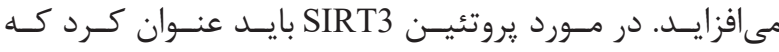

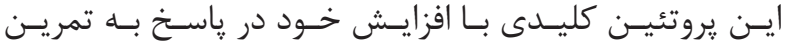

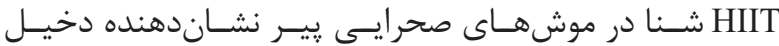

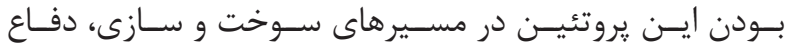

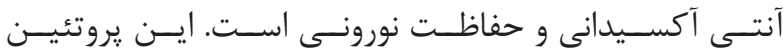

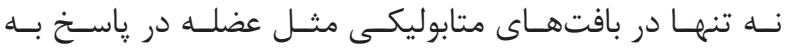

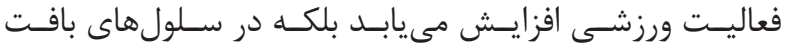

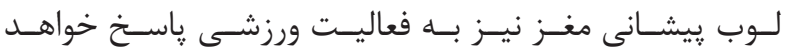

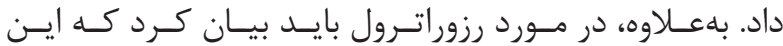

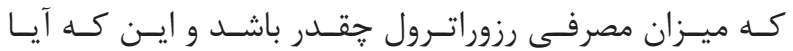

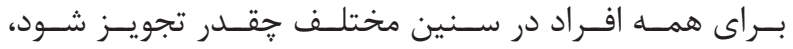

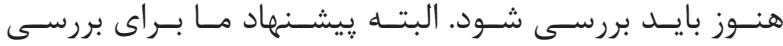

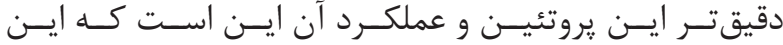

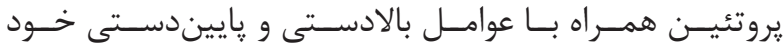

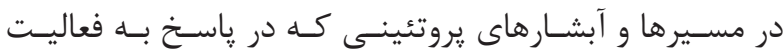

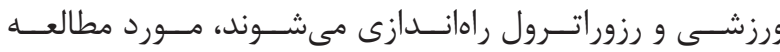

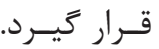

$$
\text { تشكر و قدردانى }
$$

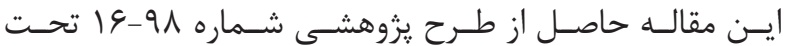

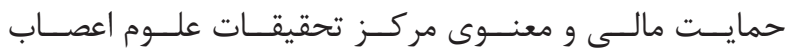

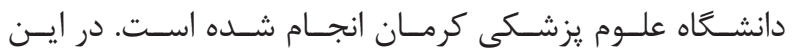

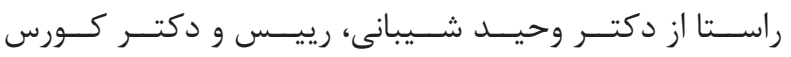

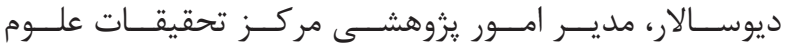

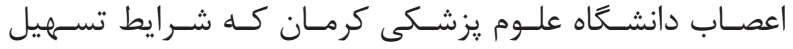

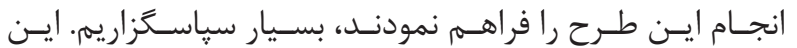

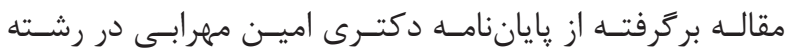

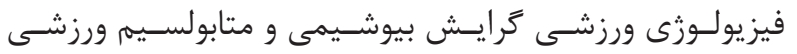

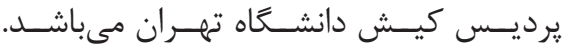

1. Cui H, Kong Y, transduction HZ-J of signal. Oxidative stress, mitochondrial dysfunction, and aging. hindawi. com, https://www.hindawi.com/archive/2012/646354/ citations/ (accessed 10 August 2020).

2. Onyango I, Lu J, Rodova M, et al. Regulation of neuron mitochondrial biogenesis and relevance to brain health. Elsevier, https://www.sciencedirect.com/science/article/ pii/S0925443909001677 (accessed 10 August 2020).

3. Trifunovic A, Larsson NG. Mitochondrial dysfunction as a cause of ageing. In: Journal of Internal Medicine. 2008, pp. 167-78.

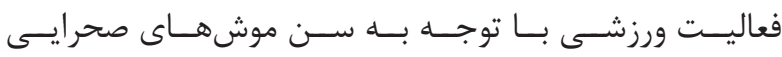

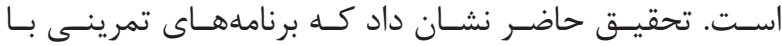

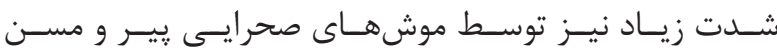

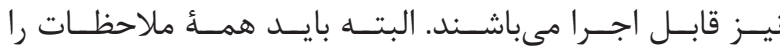

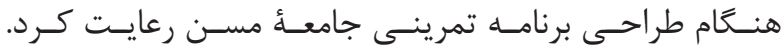

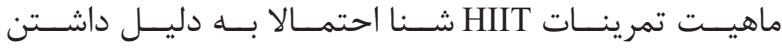

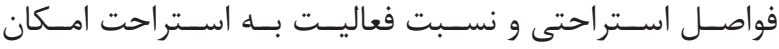

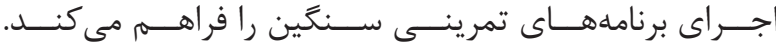

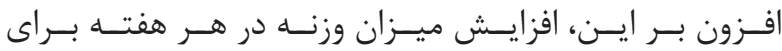

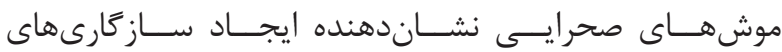

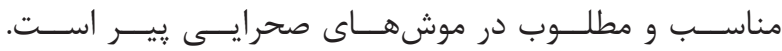

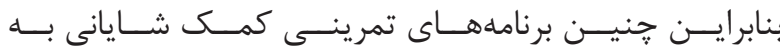

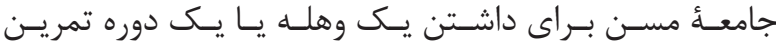

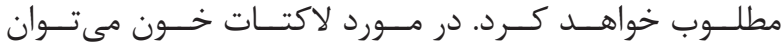

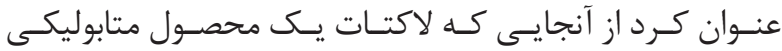

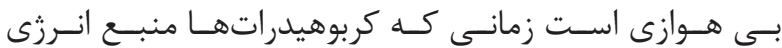

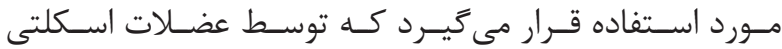

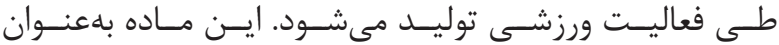

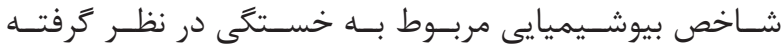

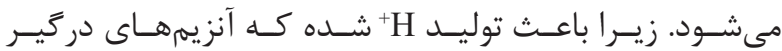

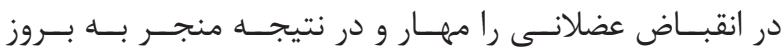

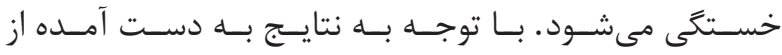

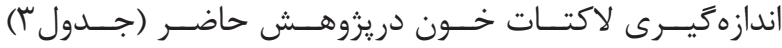

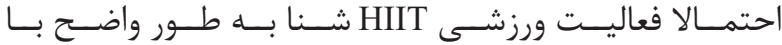

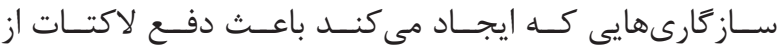

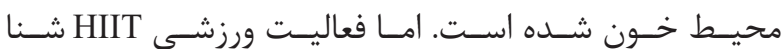

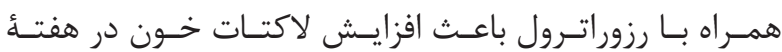

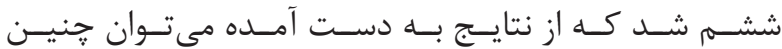

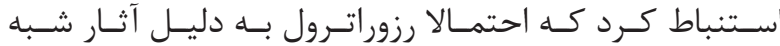

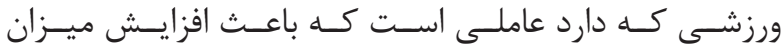

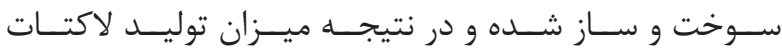

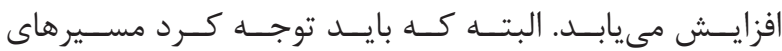

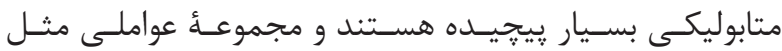

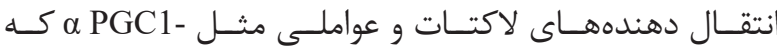

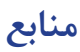

4. Jones JM, Datta P, Srinivasula SM. Loss of Omi mitochondrial protease activity causes the neuromuscular disorder of mnd2 mutant mice, www. nature.com/nature (2003, accessed 10 August 2020).

5. Genetics DW-T in, 1989 undefined. Mitochondrial DNA mutations and neuromuscular disease. Elsevier, https://www.sciencedirect.com/science/article/ pii/016895258990005X (accessed 10 August 2020).

6.LowellBB, Shulman GI.MitochondrialDysfunctionand Type 2 Diabetes. Proc Natl Acad Sci USA 2003; 30: 5050. 
7. Saxena R, Bakker P de, Singer K. Comprehensive association testing of common mitochondrial DNA variation in metabolic disease. Elsevier, https://www.sciencedirect.com/science/article/pii/ S0002929707600056 (accessed 10 August 2020).

8. Mercer JR. Suboptimal nutrition programs offspring metabolic health View project Mitochondrial bioenergetics and therapeutic intervention in cardiovasculardisease.Elsevier.Epubaheadofprint 2013.

9. Hall AR, Burke N, Dongworth RK. Mitochondrial fusion and fission proteins: Novel therapeutic targets for combating cardiovascular disease. British Journal of Pharmacology 2014; 171: 1890-906.

10. Barbosa MC, Grosso RA, Fader CM. Hallmarks of aging: An autophagic perspective. Frontiers in Endocrinology; 10. Epub ahead of print 2019.

11. Sun N, Youle RJ, Finkel T. The Mitochondrial Basis of Aging. Molecular Cell 2016; 61: 654-66.

12. German NJ, Haigis MC. Sirtuins and the Metabolic Hurdles in Cancer. CurrentBiology 2015;25: R569-R583.

13. Sebastián C, Kyle Satterstrom F, Haigis MC, et al. From Sirtuin Biology to Human Diseases: An Update *. J Biol Chem 2012; 287: 42444-452.

14. Yang W, Nagasawa K, Münch C. Mitochondrial sirtuin network reveals dynamic SIRT3dependent deacetylation in response to membrane depolarization. Elsevier, (accessed 11 August 2020).

15. Hirschey M, Shimazu T, Goetzman E. SIRT3 regulates mitochondrial fatty-acid oxidation by reversible enzyme deacetylation. nature.com, https:/www.nature com/articles/nature08778 (accessed 11 August 2020).

16. Winnik S, Auwerx J, DS-E heart. Protective effects of sirtuins in cardiovascular diseases: from bench to bedside. academic.oup.com, https:// ac ademic.oup.com/eurheartj/article-abs tract/36/48/3404/2465994 (accessed 11 August 2020).

17. Kincaid B, Bossy-Wetzel E. Forever young: SIRT3 a shield against mitochondrial meltdown, aging, and neurodegeneration. Frontiers in Aging Neuroscience; 5. Epub ahead of print 2013. 18. Jeninga E, Schoonjans $\mathrm{K}$, Oncogene JA. Reversible acetylation of PGC-1: connecting energy sensors and effectors to guarantee metabolic flexibility. nature.com, https://www.nature.com/ articles/onc2010206 (accessed 11 August 2020).

19. Kong X, Wang R, Xue Y. Sirtuin 3, a new target of PGC-1 $\alpha$, plays an important role in the suppression of ROS and mitochondrial biogenesis. PLoS One; 5. Epub ahead of print 2010.

20. Palacios O, Carmona J, Michan S. Diet and exercise signals regulate SIRT3 and activate AMPK and PGC-1 $\alpha$ in skeletal muscle. ncbi. nlm.nih.gov, https://www.ncbi.nlm.nih.gov/pmc/ articles/pmc2815736/ (accessed 11 August 2020).

21. Shi T, Wang F, Stieren E. SIRT3, a Mitochondrial Sirtuin Deacetylase, Regulates Mitochondrial Function and Thermogenesis in Brown Adipocytes*. ASBMB. $\quad$ DOI: $\quad 10.1074 /$ jbc.M414670200.

22. Pillai VB, Sundaresan NR, Kim G. Exogenous NAD Blocks Cardiac Hypertrophic Response via Activation of the SIRT3-LKB1-AMP-activated Kinase Pathway *. NUMBER 5 J Biol Chem 2010; 285: 3133.

23. Ahn B, Kim H, Song $\mathrm{S}$. A role for the mitochondrial deacetylase Sirt3 in regulating energy homeostasis. Natl Acad Sci, https://www.pnas.org/ content/105/38/14447.short (accessed 12 August 2020).

24.LombardDB, TishkoffDX,BaoJ.Mitochondrialsirtuins in the regulation of mitochondrial activity and metabolic adaptation. Handb Exp Pharmacol 2011; 206: 163-88.

25. Sidorova-Darmos E, Sommer R, Eubanks JH. The role of SIRT3 in the brain under physiological and pathological conditions. Front Cell Neurosci; 12. Epub ahead of print 25 July 2018.

26. Hallows W, Lee S, National JD-P. Sirtuins deacetylate and activate mammalian acetyl-CoA synthetases. Natl Acad Sci, https://www.pnas.org/ content/103/27/10230.short (accessed 12 August 2020).

27. Gurd B, Holloway G, Yoshida Y. In mammalian muscle, SIRT3 is present in mitochondria and not in the nucleus; and SIRT3 is upregulated by chronic muscle contraction in an adenosine. Elsevier, https://www.sciencedirect.com/science/article/pii/ S0026049511003180 (accessed 12 August 2020). 28. Nisoli E, Tonello C, Cardile. Calorie restriction 
promotes mitochondrial biogenesis by inducing the expression of eNOS. science.sciencemag.org.

29. Cohen H, Miller C, Bitterman K. Survival by Inducing the SIRT1 Deacetylase Calorie Restriction Promotes Mammalian Cell. science.sciencemag.org. Epub ahead of print 2010. DOI: 10.1126/science.1099196.

30. Hirschey M, Shimazu T, Jing E. SIRT3 deficiency and mitochondrial protein hyperacetylation accelerate the development of the metabolic syndrome. Elsevier, (accessed 12 August 2020).

31. Haigis M, Mostoslavsky R, Haigis K. SIRT4 inhibits glutamate dehydrogenase and opposes the effects of calorie restriction in pancreatic $\beta$ cells. Elsevier, (accessed 12 August 2020).

32. Cheng A, Yang Y, Zhou Y, et al. Mitochondrial SIRT3 mediates adaptive responses of neurons to exercise and metabolic and excitatory challenges. Elsevier, (accessed 12 August 2020).

33. Zhang H, Schools G, Lei T, etal. Resveratrolattenuates early pyramidal neuron excitability impairment and death in acute rat hippocampal slices caused by oxygenglucose deprivation. Elsevier, (accessed 12 August 2020).

34. Karuppagounder S, Pinto J, Xu H. Dietary supplementation with resveratrol reduces plaque pathology in a transgenic model of Alzheimer's disease. Elsevier, (accessed 12 August 2020).

35. Timmers S, Konings E, Bilet L. Calorie restrictionlike effects of 30 days of resveratrol supplementation on energy metabolism and metabolic profile in obese humans. Elsevier, (accessed 12 August 2020).

36. Menzies KJ, Singh K, Saleem A. Sirtuin 1-mediated Effects of Exercise and Resveratrol on Mitochondrial Biogenesis *. ASBMB. Epub ahead of print 2013.

37. Bagul PK, Katare PB, Bugga P, et al. SIRT-3 Modulation by Resveratrol Improves Mitochondrial Oxidative Phosphorylation in Diabetic Heart through Deacetylation of TFAM. mdpi.com.

38. Zanto TP, Gazzaley A. Aging of the frontal lobe. In: Handbook of Clinical Neurology. Elsevier B.V., 2019, pp. 369-89.

39. Wong DW, Soga T, Parhar IS. Aging and chronic administration of serotonin-selective reuptake inhibitor citalopram upregulate Sirt4 gene expression in the preoptic area ofmale mice. FrontGenet; 6. Epubahead of print 2015.

40. Amirazodi F, Mehrabi A, Amirazodi M. The Combination Effects of Resveratrol and Swimming HIIT Exercise on Novel Object Recognition and Open-field Tasks in Aged Rats. Exp Aging Res 2020; 46: 336-58.

41. Walsh RN, Cummins RA. The Open-Field Test: A Critical Review, https://psycnet.apa.org/ record/1976-27066-001 (accessed 12 August 2020).

42. Ramos-Filho D, Chicaybam G, De-Souza-Ferreira E. High intensity interval training (HIIT) induces specific changes in respiration and electron leakage in the mitochondria of different rat skeletal muscles. PLoS One; 10. Epub ahead of print 29 June 2015.

43. Shafiee A, Gaeini A, MS-J of A. The effect of eight week of high intensity interval training on expression of mir-210 and ephrinA3 mRNA in soleus muscle healthy male rats. jams.arakmu.ac.ir, http://jams.arakmu.ac.ir/ article-1-2770-en.html (accessed 12 August 2020).

44. Iman Fathi, Maryam Noorshahi. Abbas Haghparast HF hoseini. Effect of eight-week aerobic continuous and high intensity interval training on levels of SIRT3 in skeletal muscle tissue of Wis tar rats. Physiol Exerc Phys Act 1394; 8: 1277-289.

45. Karvinen S, Silvennoinen M, Vainio P. Effects of intrinsic aerobic capacity, aging and voluntary running on skeletal muscle sirtuins and heat shock proteins. Elsevier. Epub ahead of print 2016.

46. Tao R, Vassilopoulos A, Parisiadou L. Regulation of MnSOD enzymatic activity by Sirt3 connects the mitochondrial acetylome signaling networks to aging and carcinogenesis. Antioxidants and Redox Signaling 2014; 20: 1646-654.

47. White AT, Schenk S. NAD +/NADH and skeletal muscle mitochondrial adaptations to exercise. American Journal of Physiology Endocrinology and Metabolism 2012; 303: 308-21.

48. Santos-Alves E, Marques-Aleixo I, RizoRoca D. Exercise modulates liver cellular and mitochondrial proteins related to quality control signaling. Life Sci 2015; 135: 124-30. 
49. Chen T, Li J, Liu J. Activation of SIRT3 by resveratrol ameliorates cardiac fibrosis and improves cardiac function via the TGF- $\beta /$ smad 3 pathway. Am J Physiol - Hear Circ Physiol 2015; 308: 424-34.

50. Hawley JA, Joyner MJ, Green DJ. Mimicking exercise: what matters most and where to next? In: Journal of Physiology. Blackwell Publishing Ltd, 2019. Epub ahead of print 2019. 51. Sun Q, Kang R, Chen K. Sirtuin 3 is required for the protective effect of Resveratrol on Manganese- induced disruption of mitochondrial biogenesis in primary cultured neurons. J Neurochem 2020; jnc. 15095.

52. Riccioni G, Gammone MA, Tettamanti G. Resveratrol and anti-atherogenic effects. International Journal of Food Sciences and Nutrition 2015; 66: 603-10.

53. Muhammad MH, Allam MM. Resveratrol and/or exercise training counteract aging-associated decline of physical endurance in aged mice; targeting mitochondrial biogenesis and function. J Physiol Sci 2018; 68: 681-88. 